\title{
SAPPO. Sistema de autoevaluación en la nube del estándar ISO/IEC 29110 para pequeñas empresas de desarrollo de software
}

\author{
Mauricio Marroquín Rosales \\ 19990535@aloe.ulima.edu.pe / Universidad de Lima.Lima, Perú
}

Recepción: 30-6-2018 / Aceptación: 20-8-2018

\begin{abstract}
RESUMEN. Este artículo tiene como objetivo proponer un nuevo sitio web en el cual las pequeñas empresas de software puedan realizar una autoevaluación en el conjunto de estándares de calidad ISO/IEC 29110, permitiéndoles la detección de deficiencias en sus prácticas de desarrollo. Adicionalmente, el sitio web propuesto incluirá contenido educativo sobre el estándar. En este artículo se revisa brevemente la problemática de las pequeñas empresas de desarrollo de software para adoptar estándares de calidad ISO/IEC 29110 y otros estándares orientados a la pequeña empresa; el estado del arte de las herramientas de autoevaluación existentes para ISO/IEC 29110; la propuesta de implementación del sitio web en sí, y, finalmente, concluimos con algunas reflexiones sobre la importancia de la difusión y la adopción de los estándares de calidad en las micro y pequeñas empresas, y cómo estos ayudan no solo a mejorar la calidad de sus productos sino también a aumentar su competitividad en el mercado internacional. El nombre de esta nueva web propuesta es Sistema de autoevaluación para pequeñas organizaciones, (SAPPO).
\end{abstract}

Palabras ClaVe: calidad, software, ciclo de vida, ISO 29110

\section{SAPPO. Cloud self-assessment system for the ISO/IEC 29110 standard aimed at small software-development companies}

Aвstract. The objective of this article is to propose a new website in which small software companies will perform self-assessments on the set of quality standards ISO/IEC 29110, allowing the detection of deficiencies in their development practices. In addition, the proposed website will include educational content about the standard. This article examines briefly the existing barriers for quality standards adoption; ISO/IEC 29110 and other small businessoriented standards; the state of the art of existing evaluation tools for ISO/IEC 29110; the implementation of the new proposed website; and finally we conclude with some reflections on the importance of the dissemination and adoption of standards of quality in micro and small enterprises, and how these not only help to improve the quality of their products but also help to increase their competitiveness in the international market. The name of this new proposal is "Self-Assessment System for Small Organizations", acronym in Spanish: SAPPO.

KEYwORDS: quality, software, lifecycle, ISO 29110 


\section{INTRODUCCIÓN}

Según O'Connor y Laporte (2017) las pequeñas empresas de desarrollo tienden a tener una visión a corto plazo y se concentran primariamente en sobrevivir y no en crecer y expandirse, y un problema común que presenta la mayoría de las empresas pequeñas es su falta de interés en la adopción de estándares de calidad, lo cual tienden a ver como algo costoso y apropiado únicamente para grandes organizaciones (Laporte, Alexandre y O’Connor, 2008). Por consiguiente, las pequeñas empresas de software tienen formas muy limitadas de ser reconocidas como empresas que producen software de calidad y, por consiguiente, quedan fuera de algunas actividades económicas (O’Connor y Laporte, 2017). Por otro lado, existen muchas iniciativas para promover los estándares de calidad de software en las pequeñas empresas de América Latina a nivel gubernamental (MINTIC, 2018; PROSOFT, 2018), a nivel de asociaciones de empresas productoras de software y esfuerzos a nivel académico (Laporte y O'Connor, 2016; Universidad de Lima, 2018). En este contexto, SAPPO es una iniciativa adicional para promover los estándares de calidad en las pequeñas empresas, específicamente el estándar ISO/IEC 29110.

El presente artículo consta de las siguientes partes: la sección 2 describirá el estándar ISO 29110 y presentará las herramientas existentes de evaluación del mismo. La implementación propuesta de la web se encuentra descrita en la sección 3. Finalmente, las conclusiones y reflexiones sobre este trabajo se exponen en la sección 4 .

\section{ESTADO DEL ARTE}

Existe una gran variedad de estándares y modelos de calidad para empresas pequeñas de desarrollo de software. Dentro de ellos, de los que están más orientados a empresas de América Latina, podríamos mencionar a Competisoft, MoProSoft, Mps.Br e ISO 29110 (Merchán, Villa y Sánchez, 2017). Por otro lado, existen modelos como CMMI-DEV, el cual no necesariamente está enfocado en América Latina pero aun así es un modelo muy utilizado (Amable, Millones y Checa, 2015). En el marco de este trabajo nos centraremos en ISO/IEC 29110 y las herramientas de autoevaluación.

\subsection{ISO 29110}

El estándar ISO 29110 está compuesto principalmente por un grupo de documentos (figura 1) llamados también "partes", siendo los más destacables los documentos que detallan lo que ISO llama "perfiles". Estos perfiles (clasificados en perfil de entrada, básico, intermedio y avanzado) describen de forma incremental tareas que deben ejecutarse en cada proceso que pertenezca al perfil. Por ejemplo, en el perfil básico de ingeniería de software se describen las tareas que deben ejecutar los procesos de gestión de proyectos y desarrollo de software. El término Pequeña 
Organización (PO) puede referirse a una pequeña empresa o a un departamento dentro de una gran organización.

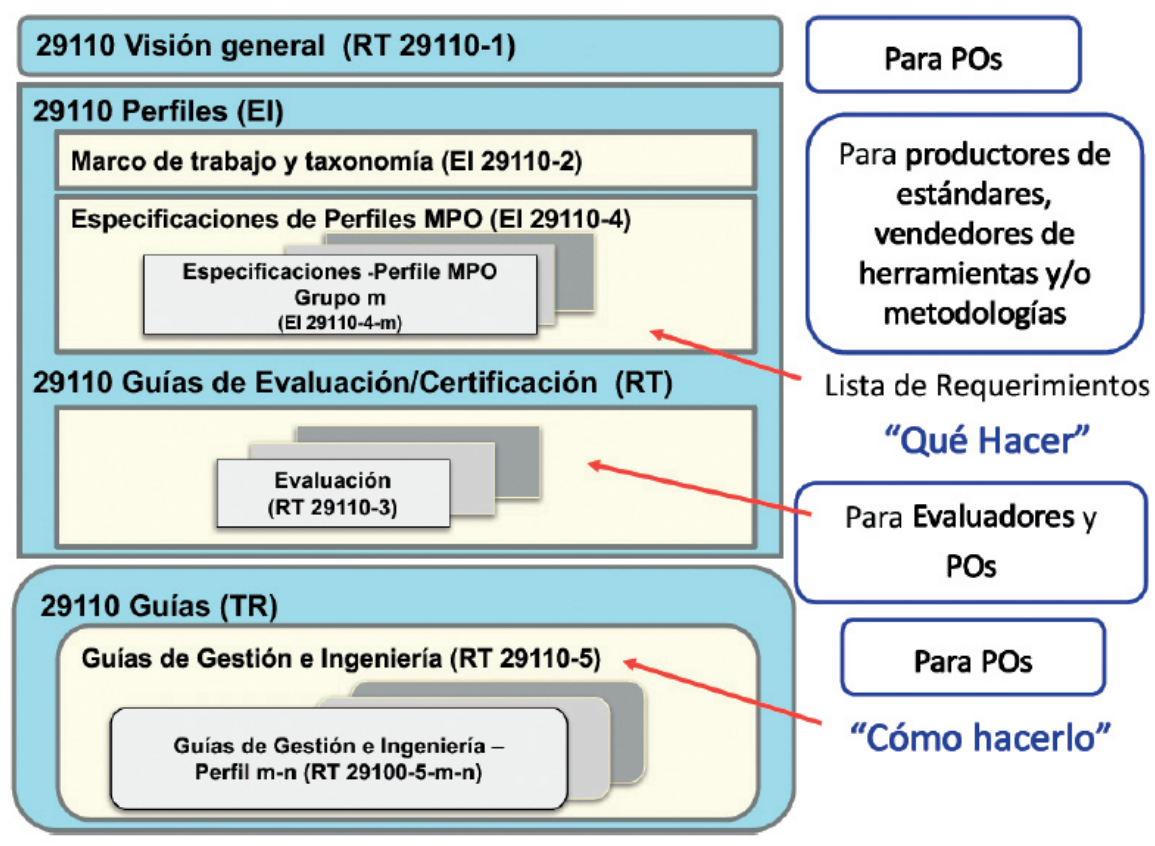

Figura 1. Estructura de ISO/IEC 29110

Fuente: Laporte, 2017

Tabla 1

Beneficios de adoptar el estándar para las pequeñas empresas

\begin{tabular}{ll}
\hline \multicolumn{1}{c}{ Internos } & \multicolumn{1}{c}{ Externos } \\
\hline Menor riesgo en el desarrollo & Incremento de competitividad \\
Mayor calidad en los productos de software & Mayor confianza y satisfacción del cliente \\
Mejor imagen institucional & \\
Mayor potencial exportador & \\
\hline
\end{tabular}

Fuente: O’Connor y Laporte, 2017 


\subsection{Herramientas de autoevaluación de ISO 29110}

Existen varias herramientas para evaluar el cumplimiento de los procesos que describe la norma ISO/IEC 29110. A continuación se describirán las herramientas que han sido encontradas en la literatura y realizando búsquedas en la web.

\subsubsection{Paquetes de despliegue: autoevaluación}

Los Paquetes de Despliegue de ISO/IEC 29110 son plantillas que facilitan la adopción del estándar, tales como plantillas de documentos técnicos y funcionales. Dentro de estas plantillas se encuentra un formato de autoevaluación (Varkoi, 2018) en formato Microsoft Excel, que proporciona una pregunta por cada tarea del perfil básico de ISO/IEC 29110. Al finalizar el cuestionario se actualiza un gráfico de barras con el porcentaje de cumplimiento. Aunque es de muy fácil uso, no provee recomendaciones y se encuentra únicamente en inglés.

\subsubsection{Encuesta de ISO 29110, CETIC}

El Centre of Excellence in Information and Communication Technologies (CETIC) es una organización de investigación en ingeniería de software y proporciona entre sus servicios en línea (www.cetic.be) una encuesta web para evaluar a las empresas en el perfil básico de ISO 29110. El cuestionario provee preguntas genéricas relacionadas al desarrollo de software y, al finalizar el cuestionario, el usuario recibe un correo electrónico con recomendaciones para cumplir mejor con el estándar. Este sistema, a diferencia del anterior, sí provee sugerencias de mejora, pero igualmente se encuentra únicamente en inglés.

\subsubsection{Herramienta de autoevaluación de ISO 29110, Upto25.net}

Upto25.net (www.upto25.net) es un sitio web de difusión de temas de calidad de software orientado a pequeñas empresas, el cual proporciona dentro de sus servicios gratuitos una herramienta de autoevaluación para el perfil básico de ISO/IEC 29110. Este cuestionario es similar al Paquete de Despliegue mencionado anteriormente, con la diferencia de que el resultado de la evaluación produce una lista con el cumplimiento de las actividades calificadas con letras ( $\mathrm{N}$ : no cumple, P: cumple parcialmente, A: cumple ampliamente, C: cumple totalmente). Este sistema provee un cuestionario en español pero no provee información gráfica y requiere que el usuario conozca modelos de evaluación para entender las calificaciones.

\subsubsection{Otras herramientas}

Existen otros trabajos relacionados, como el de Campos (2013) que traduce y adapta el Paquete de Despliegue de Autoevaluación al español, pero lamentablemente no existe un repositorio 
donde se pueda ubicar el archivo Excel mencionado. Otro trabajo destacable es el de Andrade (2017) que propone una herramienta de evaluación en la web del perfil básico con dashboards y sugerencias, pero tampoco provee la ubicación de la herramienta, dando a entender que solamente es un trabajo teórico.

\section{Propuesta}

\subsection{Comparación de herramientas}

Con las herramientas de autoevaluación mencionadas anteriormente se procedió a realizar una comparación de sus características que se puede apreciar en la tabla 2. De esto podemos resaltar que, aunque existe una herramienta en español, solo se limita a evaluar el perfil básico, no muestra gráficas ni sugerencias y los resultados no son tan sencillos de interpretar como los de las otras herramientas.

Tabla 2

Comparación de herramientas de autoevaluación

\begin{tabular}{lllllll}
\hline Medio & Organización & Idiomas & Perfiles & Gráficas & Sugerencias & Dificultad \\
\hline Excel & ISO & Inglés & Básico & Sí & No & Media \\
Web & CETIC & Inglés & Básico & No & Sí & Baja \\
Web & Upto25 & Español/inglés & Básico & No & No & Alta \\
\hline
\end{tabular}

Elaboración propia

\subsection{SAPPO}

Por lo anteriormente mencionado se considera crear un nuevo sitio web permanente en la nube, en español, que permita la evaluación en los diferentes perfiles del estándar ISO/IEC 29110 de una forma sencilla, visual y con un motor de sugerencias, permitiéndole un entendimiento al usuario de las mejoras específicas que debe realizar en sus procesos. Complementariamente, el sitio web contendrá material educativo en español e inglés, accesible para personas que no tienen necesariamente conocimientos en estándares de calidad, con ejemplos didácticos y evitando el uso excesivo de tecnicismos. 


\subsubsection{Arquitectura}

El Cloud Computing posee muchas ventajas en comparación con los servidores on-premise, tales como la ausencia de requerimientos de hardware y software, menor requerimiento de licencias, bajos costos de mantenimiento, flexibilidad y acceso mundial (Tabrizi e Ibrahim, 2017; Müller, Holm y Søndergaard, 2015; Avram, 2014; Xue y Xi, 2016). Adicionalmente las plataformas ofrecen muchos servicios complementarios (autenticación, mensajería, entre otros servicios) los cuales facilitan la implementación de aplicaciones y sitios web. Considerando estos factores, el nuevo sitio se implementará sobre una plataforma en la nube. Una arquitectura referencial propuesta para el nuevo sitio se muestra en la figura 2. No se detallará el aspecto técnico puesto que está fuera del alcance de este artículo.

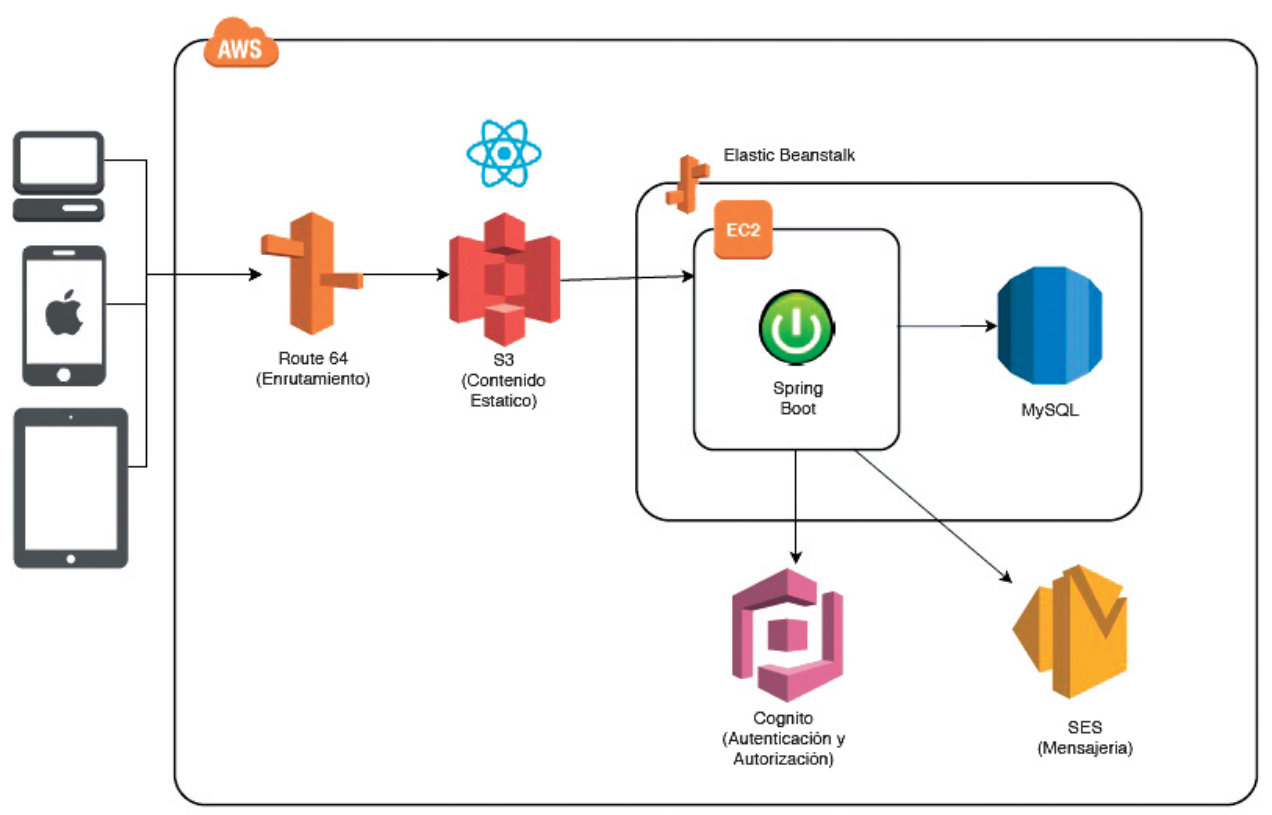

Figura 2. Arquitectura propuesta

Elaboración propia

\subsection{Funcionamiento}

La web será accesible desde el dominio www.mejoratucalidaddesoftware.com vía protocolo seguro. En caso de que se trate se acceder por protocolo inseguro, automáticamente será redirigido al seguro. Se eligió este dominio y extensión por su facilidad para ser recordados.

La página será diseñada para permitir una navegación intuitiva y el diseño se adaptará al tamaño de la pantalla del dispositivo donde será visualizado. La página principal contendrá un video introductorio de un experto en el estándar y una sección de preguntas frecuentes. El 
contenido principal está dividido en la descripción, estructura y utilización del estándar. El texto está elaborado de tal forma que los términos importantes del estándar están resaltados para una mejor identificación (figura 3).
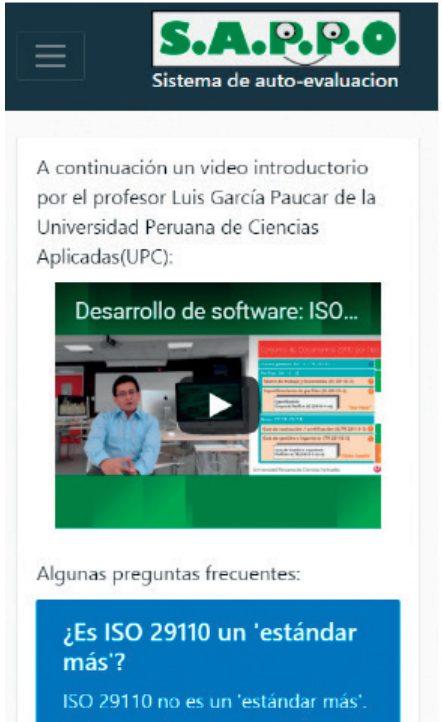

Algunas preguntas frecuentes:

\section{¿ES ISO 29110 un 'estándar más'? \\ ISO 29110 no es un 'estándar más'}

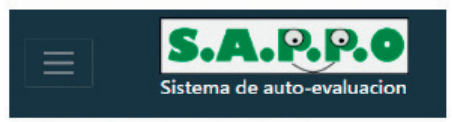

¿Que es ISO $29110 ?$

¿Como esta estructurado?

¿Como se usa?

Vinculos de interes

ISO 29110 es fruto del trabajo del comité SC7 de la Organización de Estándares Internacionales (ISO).

ISO reconoce la importancia de las pequeñas organizaciones y su contribución a la sociedad y por esto decide crear un estándar especificamente para ellas. El estándar hace énfasis psnerial en 2 áreas.

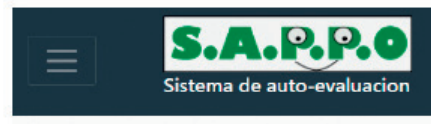

sugiere que utilices como reterencia el Perfil de entrada. Si tienes más personas que una startup, usas el Porfil básico como referencia. En la siguiente sección veras que perfil es el más adecuado dependiendo de ciertos criterios.

Continuando con la explicación, cada perfil hace referencia a la implementación de varios procesos En el Perfil de entrada y en el Perfil básico veras que se hace referencia al proceso Gestión del Proyectos y al proceso Implementación de Software.

El propósito del proceso de

Gestion del Proyecto es establecer y llevar a cabo de manera sistemática las tareas del proyecto de implementación de Software, las cuales permiten cumplir con

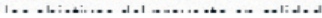

Figura 3. Pantallas home, contenido y términos

Elaboración propia

El sistema de evaluación fue diseñado bajo la premisa de que fuera fácil de usar en todos los dispositivos en donde se visualice la web. Es por esto que se utilizan botones grandes en lugar de hipervínculos o botones de radio para elegir las opciones durante la evaluación. El flujo de evaluación está dividido en cinco pasos (figura 4):

- Instrucciones.

- Datos de la empresa a evaluar: nombre, número de empleados, ¿certificación futura?

- Selección del perfil.

- Preguntas.

- Resultados de la evaluación y sugerencias de mejora. 


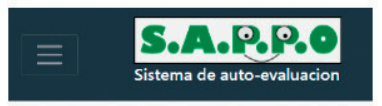

\section{Elija el tipo de perfil}

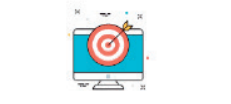

PERFIL DE ENTRADA Dirigido a POs típicamente desarrollando proyectos de 6

personas-mes o

emprendimientos.

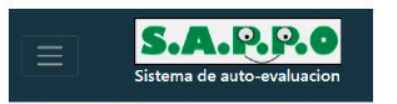

$1 / 40$

¿Se revisa el

\section{Enunciado de}

Trabajo con el

equipo?

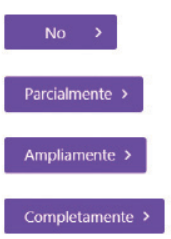

(1)

Puedes encontrar más

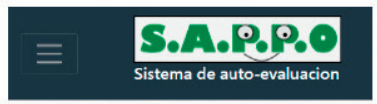

\section{Proceso de \\ Gestión de \\ Proyectos}

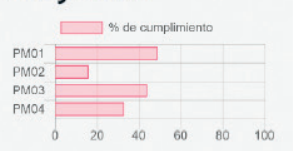

\section{Proceso de Implementación \\ de Software}

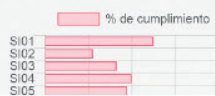

Figura 4. Pantallas elegir perfil, opciones y resultados

Elaboración propia

La metodología de evaluación utilizada será la propuesta en los Paquetes de Despliegue (Varkoi, 2018) para la autoevaluación en el perfil básico, pero se generalizará para aplicarla a los otros perfiles. Otro cambio importante es que se variará el estilo del cuestionario, realizando preguntas concretas sobre cada actividad en un lenguaje natural (tabla 3).

Tabla 3

Ejemplos de preguntas

\begin{tabular}{|l|l|}
\hline ISO 29110 & SAPPO \\
\hline PM.1.1 Revisar el enunciado de trabajo. & $\begin{array}{l}\text { ¿Se revisa el enunciado de trabajo con el } \\
\text { equipo? }\end{array}$ \\
\hline $\begin{array}{l}\text { PM.1.2 Definir con el cliente las instrucciones } \\
\text { de entrega para cada uno de los entregables } \\
\text { especificados en el enunciado de trabajo. }\end{array}$ & $\begin{array}{l}\text { ¿Se definen con el cliente las instrucciones } \\
\text { de entrega para cada uno de los entregables } \\
\text { especificados en el enunciado de trabajo? }\end{array}$ \\
\hline
\end{tabular}

Elaboración propia 


\section{Conclusiones}

Se concluye que este trabajo ofrece una herramienta que supliría las limitaciones de las herramientas de autoevaluación existentes, facilitando a las empresas la detección de deficiencias en sus procesos de desarrollo y permitiendo la adopción del estándar ISO/IEC 29110 y de buenas prácticas de desarrollo de software en general. También se concluye que, aunque las iniciativas para promover los estándares de calidad en las pequeñas empresas son muy buenas, es necesario continuar con estas iniciativas puesto que las pequeñas empresas son un actor económico importante en la sociedad.

Como reflexión final consideramos importante promover un cambio cultural en las pequeñas empresas, desde dentro y desde fuera, para orientarlas a darle importancia a los estándares de calidad y sensibilizarlas sobre el valor que otorgan a la organización y a sus clientes.

\section{REFERENCIAS}

Amable, M., Millones, R., y Checa, R. (2015). Calidad de software en las mypes productoras de software en Lima. En: Memoria COMTEL (pp. 133-139). Lima: Universidad Inca Garcilaso de la Vega.

Andrade, A. (2017). Ferramenta web para autoavaliação de aderência à norma ISO/IEC. Tesis de bachillerato. Universidade Federal de Santa Catarina.

Avram, M.-G. (2014). Benefits of cloud computing: Literature review in a maturity model perspective. Procedia Technology 12, pp. 529-534.

Campos, Y. (2013). Desarrollo de una herramienta de auto-evaluación para el cumplimiento de ISO/IEC 29110: 5-1-2. Tesis de maestría. Universidad Nacional Autónoma de México.

Laporte, C. Y., Alexandre, S., y O’Connor, R. V. (2008). A Software Engineering Lifecycle Standard for Very Small Enterprises. En: Proceedings of EuroSPI 16, pp. 129-141. Heidelberg: Springer.

Laporte, C. Y., y O’Connor, R. V. (2016). Software process improvement in industry in a graduate software engineering curriculum. Software Quality Professional Journal 18(3).

Merchán, L., Villa, L. B., y Sánchez, N. J. (junio de 2017). Guía de recomendación para la selección de un modelo de calidad para la mejora de procesos de software (SPI). Revista Ciencias Estratégicas 25(37), 25-51.

Ministerio de Tecnología de la Información y las Comunicaciones-MINTIC (2018). Con apoyo del MINTIC 100 empresas TI se han certificado en modelos de calidad. Portal del Ministerio de Tecnologias de la Información y las Comunicaciones, Colombia. Recuperado de https://www.mintic.gov.co/portal/604/w3-article-14174.html 
Müller, S. D., Holm, S. R., y Søndergaard, J. (2015). Benefits of cloud computing: Literature review in a maturity model perspective. Communications of the Association for Information Systems 37, pp. 851-878.

O'Connor, R. V., y Laporte, C. Y. (2017). The Evolution of the ISO/IEC 29110 Set of Standards and Guides. International Journal of Information Technologies and Systems Approach 10(1), 1-21.

Programa para el Desarrollo de la Industria del Software-PROSOFT (2018). Programa para el desarrollo de la industria del software. México. Recuperado de https://prosoft. economia.gob.mx/

Tabrizi, S. S., y Ibrahim, D. (2017). A Review on Cloud Computing and Internet of Things. World Academy of Science, Engineering and Technology International Journal of Computer and Information Engineering 11(4).

Universidad de Lima (2018). Workshop Internacional. Introducción a la norma ISO/IEC 29110. Recuperado de http://fresno.ulima.edu.pe/sf/sf_bd6500.nsf/default/ 6B3E8 458A1ECA34305257A64006D6E3A/\$file/Curso_ISO_29110.pdf

Varkoi, T. (2018). Deployment Package Self-Assessment Basic profile. Recuperado de http:// profs.etsmtl.ca/claporte/english/vse/deploy-pack/DP-Self-Assessm ent-V1.2.doc

Xue, C. T. S., y Xi, F. T.W. (2016). Benefits and challenges of the adoption of cloud computing in business. International Journal on Cloud Computing: Services and Architecture (IJCCSA) 6(6).

\section{BibLIOGRAFÍA}

Abran, A., Moore, J. W., Bourque, P., y Dupuis, R. (2004). Guide to the software engineering body of knowledge. New York: IEEE Computer Society Press.

Laporte, C. Y. (2017). La implementación de la norma ISO/IEC 29110. Guias de gestión e ingeniería para las organizaciones pequeñas. Recuperado de https://www.gtcsas.com/ wp-content/uploads/2017/03/Colombia_March_2017_CL0.pdf

Laporte, C. Y., Alexandre, S., y Renault, A. (2008). The Application of International Software Engineering Standards in Very Small Enterprises. Software Quality Professional 10(3). 